\title{
Distribuição espacial da granulometria, cor e carbono orgânico do solo ao longo de um transecto em microbacias na Amazônia meridional
}

\author{
Léo Adriano $\mathrm{CHIG}^{1}$, Eduardo Guimarães $\mathrm{COUTO}^{1}$, João Paulo NOVAES FILHOํㅜ, Luiz Carlos Mattos \\ RODRIGUES ${ }^{1}$, Mark S. JOHNSON${ }^{1}$, Oscarlina Lúcia dos Santos WEBER ${ }^{1}$
}

\begin{abstract}
RESUMO
O objetivo deste estudo foi determinar, em nível de detalhe, a distribuição e a variabilidade espacial da argila, do índice de avermelhamento e do carbono orgânico do solo, ao longo de transecto que cruza classes pedológicas distintas dentro de quatro microbacias, sob floresta tropical em Juruena (MT). Para isso, foram selecionados 34 pontos e coletadas amostras de solo nas profundidades de 0 a $20 \mathrm{~cm}$ e 40 a $60 \mathrm{~cm}$. Os dados foram analisados por estatística descritiva e geoestatística. As maiores variabilidades espaciais ocorreram dentro da classe do Latossolo Vermelho-Amarelo Distrófico. Estes ocorrem nas elevaçōes superiores a $280 \mathrm{~m}$, enquanto os Plintossolos e Argissolos (com caráter plíntico) têm ocorrência restrita às áreas com altimetrias menores. Ao longo do transecto há maior variabilidade nos atributos índice de avermelhamento e argila; as áreas com piores condiçōes de drenagens apresentaram menor variação espacial e menores índice de avermelhamento, isto é, maior homogeneidade da cor dos horizontes diagnósticos.
\end{abstract}

PALAVRAS-CHAVE: Índice de avermelhamento, variabilidade espacial, funçōes de pedotransferência.

\section{Spatial distribution of soil texture, color and soil organic carbon across a headwater transect in the southern Amazon}

\section{ABSTRACT}

The study was conducted in forested headwater catchments near Juruena, Mato Grosso with the objective of determining the spatial distribution of soil physical characteristics including clay content, redness ratio, and soil organic carbon along a transect that encompassed distinct soil classes. Soil samples from $0-20 \mathrm{~cm}$ and $40-60 \mathrm{~cm}$ depths were collected from 34 points. Variables were analyzed using descriptive statistics and geostatistics. The parameters analyzed by ordinary kriging demonstrated that the highest spatial variability occurred within the soil class Typic Kandiustox. In the study area, Plinthustults and Ultisols (with plinthic characteristics) were only found to occur in landscape positions less than $280 \mathrm{~m}$ above sea level; Oxisols were found exclusively above 280 m.a.s.l. The redness ratio and clay content were the soil parameters with the highest spatial variability across the transect. Poorly drained areas (demonstrated by lower values of redness ratio) demonstrated the lowest degree of spatial variability, and correspondingly, the highest consistency of color within the diagnostic horizons.

KEY-WORDS: Redness ratio, spatial variability, pedotransfer functions

Departamento de Solo e Extensão Rural/FAMEV/UFMT leochig@gmail.com, couto@cpd.ufmt.br, jpnovaes@terra.com.br, mattosr@cpd.ufmt.br, mark.johnson@ubc.ca, oscsanwb@ufmt.br. 


\section{INTRODUÇÃO}

A grande diversidade geológica existente na Amazônia, aliada ao relevo diferenciado, resultou na formação das mais variadas classes de solo, sob a influência das altas temperaturas e precipitações, características do clima tropical úmido. Contudo, a fertilidade natural dos solos, em geral, é baixa e depende dos nutrientes que recebe da floresta (Loureiro, 2002).

Os solos predominantes são Latossolos e Argissolos (FAO, 1988; EMBRAPA, 2006). Destas classes mais comuns, a primeira é caracterizada por solos mais profundos e intemperizados; a segunda, por ter subsolo menos poroso, com textura mais argilosa do que o horizonte superficial. Sombroek (1984) destaca que estes solos são predominantes, quimicamente pobres, constituídos por fração de argila com baixa atividade, cuja maior parte dos nutrientes disponíveis para as plantas está relacionada com a matéria orgânica. Esta, se encontra concentrada nas camadas superficiais do solo, varia de 1 a $3 \%$ e se concentra a até $30 \mathrm{~cm}$ de profundidade, dependendo da textura do solo e da cobertura vegetal (Novaes Filho et al., 2007b).

As informações a respeito da distribuição de solos na Amazônia são fundamentadas, principalmente em levantamentos generalizados de solos. Poucos são os trabalhos ao nível de detalhe ou semidetalhe, o que permite concluir que, não obstante a monotonia da paisagem, os tipos de solos amazônicos são bastante variáveis (Novaes Filho et al., 2007b).

O solo, segundo Oliveira et al. (2000), apresenta como característica marcante a sua heterogeneidade. Na paisagem natural, ocorre ampla variação das propriedades morfológicas, físicas, químicas e mineralógicas, tanto na posição horizontal como na vertical, resultante dos processos pedogenéticos. $\mathrm{O}$ conhecimento dessa variação é importante no levantamento, na sua classificação, na avaliação de sua fertilidade e, principalmente, para o desenvolvimento de esquemas de amostragem mais adequados. Os enfoques da variabilidade espacial dos atributos do solo, por meio da krigagem ordinária, permitem estimar, apropriadamente, os valores de atributos em áreas não amostradas, além de direcionar projetos futuros com pesquisas de amostragens de solo (Vieira, 2000).

Em estudos pedológicos no sul do Estado de Mato Grosso, Couto et al. (2000) estimaram, por meio da aplicação da krigagem ordinária, os valores de potássio trocável e de argila nos horizontes A e B de solos ao longo de transectos que cruzaram classes pedológicas distintas. Isso permitiu identificar os locais onde as amostragens devem se concentrar nos futuros trabalhos de levantamentos pedológicos e evidencia, assim, a importância de se levar em consideração a dependência espacial neste tipo de pesquisa.
Importantes descobertas na ciência do solo ocorreram em razão do conhecimento da estrutura da dependência espacial. Cambardella et al. (1994) verificaram a existência de semelhanças nos padrōes de variabilidade espacial entre alguns atributos de solo em locais diferentes. Isso fortalece a possibilidade da extrapolação das relaçóes medidas em uma área para outros locais não amostrados, dentro de uma mesma bacia hidrográfica ou em escala regional.

O objetivo deste trabalho foi detalhar a variabilidade espacial do conteúdo de argila e carbono orgânico, além do índice de avermelhamento de solos ao longo de transecto que atravessa classes pedológicas distintas em quatro microbacias localizadas município de Juruena, região noroeste do Estado de Mato Grosso.

\section{MATERIAL E MÉTODOS}

A área de estudo está localizada na Fazenda Rohsamar (coordenadas geográficas: 10²8’ Se 58²6’ WGr.), município de Juruena, região noroeste do Estado de Mato Grosso, distante $920 \mathrm{~km}$ da capital Cuiabá. A cobertura vegetal existente na região é classificada, segundo o IBGE (1990), como Floresta Ombrófila Densa de formação submontana. É denominada floresta de terra firme, caracterizada por apresentar árvores de médio e grande porte, com grande número de palmeiras.

A região possui clima tropical quente e úmido (Aw), pela classificação de Köppen (BRASIL, 1980), com temperatura média anual de $24^{\circ} \mathrm{C}$ e precipitação total durante o ano de 2004 de $2.379 \mathrm{~mm}$ (Johnson et al., 2004). O regime de umidade do solo é caracterizado como ústico, pois o período seco atinge mais de 90 dias (Van Wambeke, 2002).

Os solos estão sobre rochas do Complexo Xingu de idade pré-cambriana. A unidade geomorfológica é a Depressão Interplanáltica da Amazônia Meridional, com declividade suave ondulada na maior parte da região e altitude entre 250 e 300 m (BRASIL, 1980).

Foram selecionados 34 pontos e coletadas amostras do solo nas profundidades de 0 a $20 \mathrm{~cm}$ (P1) e 40 a $60 \mathrm{~cm}$ (P2), perfazendo um total de 68 amostras. O esquema de amostragem foi disposto num transecto no sentido oeste-leste (Figura 1), em quatro microbacias sob vegetação de floresta não perturbada, próximas entre si e com grande variação de solos, segundo Novaes Filho et al. (2007b). As amostras foram secas em estufa de circulação de ar forçada a $70^{\circ} \mathrm{C}$ por 42 horas; após a secagem, foram peneiradas com malha de 2 $\mathrm{mm}$ e devidamente acondicionadas e identificadas. A análise granulométrica foi realizada pelo método densimétrico, conforme descrito pela EMBRAPA (1997).

As leituras da cor do solo (amostras secas) foram feitas em laboratório, com a utilização de colorímetro Minolta, modelo CR-400, que interpreta a cor a partir da energia refletida na 
região visível. O equipamento determina a cor no sistema de coordenadas XYZ e, em seguida, foi obtida a cor na notação Munsell. As medidas de cor feitas pelo colorímetro podem ser obtidas em intervalos intermediários que não são apresentados na carta de Munsell (1975), conferindo maior precisão e exatidão a essas determinaçôes. Uma utilização imediata das cores, segundo Torrent \& Barròn (1993), é a determinação do índice de avermelhamento (IAV). Este parâmetro é uma expressão que combina os três componentes da cor (matiz, valor e croma) (eq. 1).

Índice de Avermelhamento $=[(10-\mathrm{H}) \times \mathrm{C}] / \mathrm{V}$

Em que: $\mathrm{H}=$ Matiz $($ matiz $=10 \mathrm{YR} \rightarrow \mathrm{H}=10 \mathrm{e}$ matiz $=10 \mathrm{R} \rightarrow \mathrm{H}=0$ ).

De acordo com os critérios de cor recomendados pela EMBRAPA (2006), foi feita correspondência entre a nomenclatura da cor (2。 nível categórico) e o índice de avermelhamento (IAV) da seguinte forma: IAV $\leq 1,8=$ solos amarelos; IAV variando de 1,9 a 3,3 = solos vermelhoamarelos; e IAV $\geq 3,4$ = solos vermelhos.

O carbono total foi determinado no analizador Multi NC 3.000 (Analytik Jena, Germany), com combustão seca (forno Eltra, $1.350^{\circ} \mathrm{C}$ ), onde todas as formas de carbono da amostra são convertidas em $\mathrm{CO}_{2}$ seguida de quantificação total desse gás (carbono total), conforme metodologia descrita pelo Soil Survey Staff (1996). Foi assumido que o teor de carbono orgânico (CO) é igual ao de carbono total, devido os solos apresentarem valores de $\mathrm{pH}$ em solução aquosa inferiores a 7,0 (indicativo de ausência de carbonatos).

A análise estatística foi realizada em duas etapas. $\mathrm{Na}$ primeira, foi empregada a estatística clássica, por meio do software SPSS 10 (2000), onde foi determinada: média, análise de variância, teste de homogeneidade da variância, coeficiente de variação $(\mathrm{CV})$, grau de dispersão $(\mathrm{GD})$, amplitude de variação, assimetria, análise de distribuição de freqüência de dados e o teste de distribuição normal. Na segunda etapa, a análise dos dados foi fundamentada na teoria das variáveis regionalizadas (geoestatística), compreendendo as funçōes do semivariograma (equação 2) e a krigagem (equação 3), com o auxilio dos softwares Gamma Design - GS+ (Robertson, 2000) e Surfer (Surfer, 2000).

O parâmetro do semivariograma, com base nas pressuposiçōes de estacionariedade da hipótese intrínseca, segundo Journel \& Huijbregts (1978) e Vieira (2000), é estimado por:

$$
\gamma(h)=\frac{1}{2 N(h)} \sum_{i=1}^{N(h)}\left[Z\left(x_{i}\right)-Z\left(x_{i}+h\right)\right]^{2}
$$

Em que: $\gamma(\mathrm{h})$ é o semivariograma estimado; $\mathrm{N}(\mathrm{h})$ é o número de pares de pontos medidos $Z(x i)$ e $Z(x i+h)$, separados por vetor $h$.
A análise da função semivariância, de acordo com Guimarães (2001), conduz às seguintes interpretaçōes: quanto mais próximos estiverem os pontos amostrados, maior é a semelhança entre eles e, portanto, menor a semivariância; e menor a dispersão e vice e versa.

De acordo com Couto \& Klamt (1999), Gonçalves et al. (1999) e Vieira (2000), a krigagem a qual representa combinação linear dos valores medidos, pode ser expressa pela equação 3:

$$
\mathrm{Z}\left(\mathrm{x}_{0}\right)=\sum_{\mathrm{i}=1}^{\mathrm{N}} \lambda_{\mathrm{i}} \mathrm{Z}\left(\mathrm{x}_{\mathrm{i}}\right)
$$

Em que: $\mathrm{Z}\left(\mathrm{x}_{0}\right)$ é o valor medido para cada local não amostrado; $\mathrm{N}$ é o número de valores medidos, $\mathrm{Z}(\mathrm{xi})$, envolvidos na estimativa; e $\lambda$ são os pesos associados a cada valor medido.

Para verificar a variabilidade destas propriedades ao longo da distância foi constituído transecto, visualizado na Figura 1. Neste transecto, foram estimados por meio de krigagem os valores dos atributos estudados a partir dos variogramas totais.

\section{RESULTADOS E DISCUSSÃO}

Ao observar a distribuição das classes de solos (Figura 1), foi verificado que as microbacias 2, 3 e 4 apresentaram predomínio de Latossolos, enquanto que a microbacia 1, apresentou maior diversidade de classes pedológicas (Argissolo Amarelo, Plintossolo, Latossolo Vermelho-Amarelo e Latossolo Vermelho). Isso, segundo Jenny (1941), demonstra a complexa

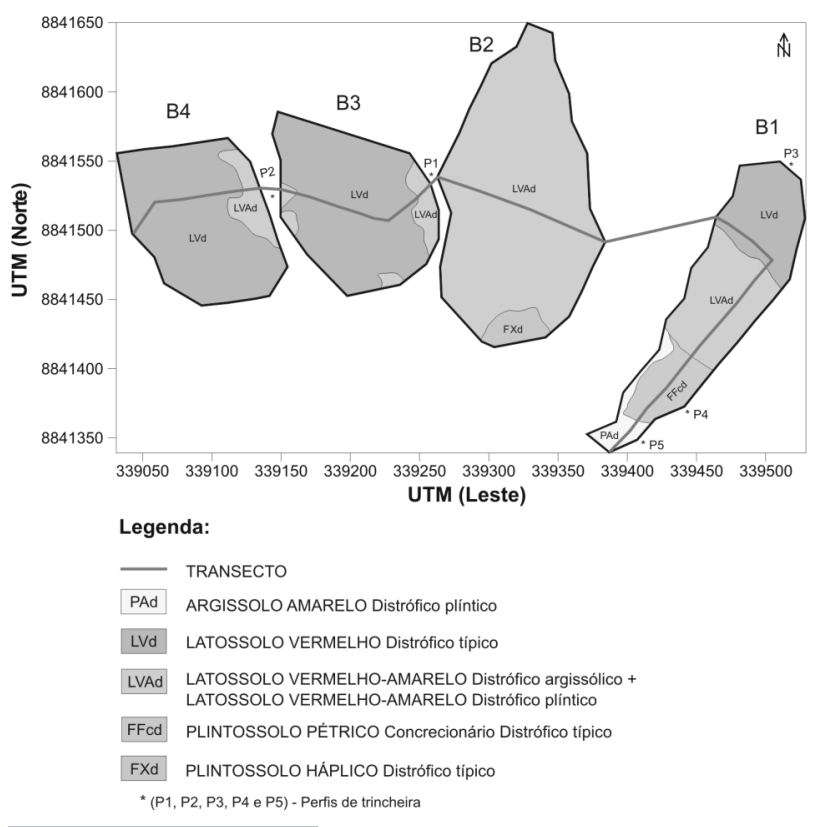

Figura 1 - Arranjo do transecto e distribuição das classes de solos nas quatro microbacias (Novaes Filho et al., 2007b). 
dinâmica dos fatores de formação de solo, caracterizada pela grande variabilidade em um pequeno espaço.

O coeficiente de variação $(\mathrm{CV})$ dos atributos estudados (Tabela 1) apresentou valores semelhantes às observaçōes de Wilding \& Drees (1983) e Novaes Filho et al. (2007a,b), o que confere maior segurança aos resultados. $\mathrm{O}$ grau de dispersão da variação da altitude ficou abaixo de $10 \%$ de acordo com a classificação de Pimentel Gomes (2000). Os teores de carbono orgânico de todas as classes pedológicas, na profundidade de 0 a $20 \mathrm{~cm}$ (P1), tiveram grau de dispersão médio, devido os coeficientes de variação estarem situados entre 10 e $20 \%$. $\mathrm{Na}$ profundidade de 40 a $60 \mathrm{~cm}$ (P2), essa variação foi de média a muito forte (11 a 58\%). O teor de argila apresentou coeficiente de variação de médio a forte em P1 (CV - 18,25 a $27,14 \%$ ) e de baixa a forte em P2 (CV - 7,54 a 25,54\%).
Enquanto que, para o índice de avermelhamento (IAV), o coeficiente de variação observado foi muito forte em P1 (CV - 44,05 a 91,62\%) e de forte a muito forte em P2 (CV $-27,49$ a $141,33 \%)$.

O teor médio de CO observado nas classes pedológicas cortadas pelo transecto, na camada superior, foi de 9,46 g. $\mathrm{kg}^{-1}$, o que é compatível com os resultados de Botschek et al. (1996) e Ruivo et al. (2002) em Latossolos Amarelos na Amazônia; porém, são bem inferiores aos teores encontrados por Laurance et al. (1999) e McGrath et al. (2001) em diferentes solos.

Os valores médios dos teores de argila e carbono orgânico não apresentaram diferenças estatísticas significativas entre as classes de solos das microbacias (Tabela 1), tanto em P1, quanto em P2. Possivelmente, isto se deve à semelhança do material de origem dos solos, onde o fornecimento de carbono

Tabela 1 - Estatística descritiva dos atributos estudados no transecto.

\begin{tabular}{|c|c|c|c|c|c|c|c|c|c|}
\hline & Classe & Min. & Max. & Amplitude & Média & & SD & CV & $\mathrm{GD}$ \\
\hline \multirow{4}{*}{$\begin{array}{l}\text { Altitude } \\
\text { (m) }\end{array}$} & PAd & 270,11 & 271,96 & 1,85 & 271,04 & B & 1,31 & 0,48 & B \\
\hline & FFcd & 274,47 & 278,90 & 4,43 & 276,52 & B & 2,23 & 0,81 & B \\
\hline & LVAd & 280,04 & 285,76 & 5,72 & 283,85 & $A$ & 1,56 & 0,55 & $B$ \\
\hline & LVd & 277,09 & 287,21 & 10,12 & 283,53 & A & 3,36 & 1,19 & B \\
\hline \multicolumn{10}{|c|}{0 a $20 \mathrm{~cm}(\mathrm{P} 1)$} \\
\hline \multirow{4}{*}{$\begin{array}{l}\text { Argila } \\
(\%)\end{array}$} & PAd & 18,10 & 26,70 & 8,60 & 22,40 & A & 6,08 & 27,14 & $F$ \\
\hline & FFcd & 25,10 & 31,70 & 6,60 & 28,55 & A & 3,31 & 11,59 & M \\
\hline & LVAd & 16,55 & 40,30 & 23,75 & 27,38 & A & 5,02 & 18,33 & $M$ \\
\hline & LVd & 25,70 & 41,05 & 15,35 & 31,35 & A & 5,72 & 18,25 & $\mathrm{M}$ \\
\hline \multirow{4}{*}{$\begin{array}{l}\text { Carbono } \\
\text { orgânico } \\
\left(\mathrm{g} \cdot \mathrm{kg}^{-1}\right)\end{array}$} & PAd & 7,20 & 8,59 & 1,39 & 7,90 & A & 0,98 & 12,41 & M \\
\hline & FFcd & 9,08 & 11,05 & 1,97 & 9,84 & A & 1,06 & 10,77 & M \\
\hline & LVAd & 7,73 & 12,96 & 5,23 & 9,75 & A & 1,52 & 15,59 & M \\
\hline & LVd & 7,93 & 12,65 & 4,72 & 10,33 & A & 1,51 & 14,62 & M \\
\hline \multirow{4}{*}{ IAV } & PAd & - & - & - & - & & - & - & - \\
\hline & FFcd & 0,00 & 3,00 & 3,00 & 1,67 & B & 1,53 & 91,62 & MF \\
\hline & LVAd & 2,00 & 7,50 & 5,50 & 3,95 & B & 1,74 & 44,05 & MF \\
\hline & LVd & 2,50 & 15,00 & 12,50 & 8,29 & A & 3,76 & 45,36 & MF \\
\hline \multicolumn{10}{|c|}{40 a $60 \mathrm{~cm}(\mathrm{P} 2)$} \\
\hline \multirow{4}{*}{$\begin{array}{l}\text { Argila } \\
\text { (\%) }\end{array}$} & PAd & 40,45 & 45,00 & 4,55 & 42,73 & $A$ & 3,22 & 7,54 & $B$ \\
\hline & FFcd & 29,60 & 49,85 & 20,25 & 41,58 & $A$ & 10,62 & 25,54 & $\mathrm{~F}$ \\
\hline & LVAd & 34,10 & 49,25 & 15,15 & 43,15 & A & 4,56 & 10,57 & M \\
\hline & LVd & 33,95 & 47,65 & 13,70 & 39,45 & A & 3,64 & 9,23 & B \\
\hline \multirow{4}{*}{$\begin{array}{l}\text { Carbono } \\
\text { orgânico } \\
\left(\mathrm{g} \cdot \mathrm{kg}^{-1}\right)\end{array}$} & PAd & 3,65 & 5,54 & 1,89 & 4,60 & A & 1,34 & 29,13 & $F$ \\
\hline & FFcd & 1,63 & 6,39 & 4,76 & 4,15 & $A$ & 2,39 & 57,59 & MF \\
\hline & LVAd & 4,26 & 7,04 & 2,78 & 5,25 & A & 0,63 & 12,00 & M \\
\hline & LVd & 4,64 & 6,90 & 2,26 & 5,24 & A & 0,59 & 11,26 & M \\
\hline \multirow{4}{*}{ IAV } & PAd & 0,00 & 3,00 & 3,00 & 1,50 & $C$ & 2,12 & 141,33 & MF \\
\hline & FFcd & 3,00 & 6,00 & 3,00 & 4,00 & $\mathrm{BC}$ & 1,73 & 43,25 & MF \\
\hline & LVAd & 6,00 & 15,00 & 9,00 & 7,42 & B & 2,55 & 34,37 & MF \\
\hline & LVd & 6,00 & 15,00 & 9,00 & 10,55 & A & 2,90 & 27,49 & $\mathrm{~F}$ \\
\hline
\end{tabular}

Classe: PAd - Argissolo Amarelo Distrófico plíntico, FFcd - Plintossolo Pétrico Concrecionário distrófico típico, LVAd - Latossolo Vermelho-Amarelo Distrófico, LVd - Latossolo Vermelho Distrófico típico; Min. - valor mínimo; Máx. - valor máximo; Médias - seguidas por mesma letra não diferem entre si, pelo teste de Tukey a 5\% de probabilidade; SD Desvio Padrão; CV - Coeficiente de Variação (\%); GD - Grau de dispersão: B (Baixo) $=$ CV $\leq 10 \%$; M (Médio) $=10 \%<C V<20 \%$; F (Forte) $=20 \%<C V<30 \%$; e MF (Muito Forte) $=\mathrm{CV}>30 \%$ (Pimentel Gomes, 2000). 
pela floresta deve ser similar entre as microbacias, evidenciando assim, a influência exercida pelos fatores de origem e biológico. Cabe ressaltar que, em estudo realizado por Rodrigues (2005) na microbacia 2, demonstrou que os materiais presentes nas diferentes posições da paisagem são alóctones (ex-situ).

Os resultados dos semivariogramas (Tabela 2) demonstraram que houve dependência espacial nas duas profundidades para os atributos estudados, o que possibilitou o uso da krigagem e a conseqüente distribuição espacial dos atributos acima citados. Os modelos do semivariograma que se ajustaram melhor aos resultados dos atributos do solo foram o esférico e o exponencial, corroborando com as afirmaçôes de McBratney \& Webster (1986), Vieira (1997) e Carvalho et al. (2002). Todos os atributos estudados apresentaram forte dependência espacial, de acordo com o critério proposto por Cambardella et al. (1994), pois as proporçôes do efeito pepita $\left(\mathrm{C}_{0}\right)$ em relação ao patamar $\left(\mathrm{C}+\mathrm{C}_{0}\right)$ foram relativamente baixos, de $2 \%$ a $21 \%$.

Para os atributos estudados, em ambas as profundidades, foram observados alcance mínimo de $136 \mathrm{~m}$ e máximo de $517 \mathrm{~m}$, os quais indicam a amplitude de correlação espacial entre as observaçóes. Isso representa a distância em que a utilização das técnicas geoestatísticas conduz a estimativas com maior precisão. Esta relação de dependência espacial implica em interação entre os dados, os quais não podem ser considerados independentes dentro do domínio de $517 \mathrm{~m}$. Foi constatado o aumento da dependência espacial nos horizontes subsuperficiais, fato este também relatado nos trabalhos de Couto \& Klamt (1999) e Lobato (2000).

A análise da distribuição das classes de solo, associada ao relevo da paisagem (Figura 2A) verificou que os horizontes B textural (Bt) ocorreram em cotas inferiores a $280 \mathrm{~m}$ de altitude, e se encontraram associados a processos de plintização, como descreveram Novaes Filho et al. (2007b). Assim, os Argissolos (com caráter plíntico) e Plintossolos foram observados na paisagem das microbacias estão em altitudes estimadas menores que $280 \mathrm{~m}$ e, os Latossolos, nas cotas superiores a essa.

Uma característica pedológica marcante nas classes de solo distribuídas ao longo das microbacias, observada na comparação das Figuras 2D e 2E, é a migração de argila para o subsolo. O processo de translocação da argila, segundo Gonzalez \& Alves (2005), ocorre pelos poros médios e grandes ou em fendas, onde há a deposição quando os poros maiores não têm continuidade e a água transloca para poros menores e deposita as partículas nas paredes dos agregados.

Quanto ao índice de avermelhamento, a classe Latossolo Vermelho Distrófico típico, caracterizada por um universo pedológico mais simples, expressou maior homogeneidade, principalmente na profundidade de $40-60 \mathrm{~cm}$, onde o efeito pigmentário da matéria orgânica é menor, pois é mais abundante no topo do solo (especialmente em Latossolos e Argissolos). Nos horizontes subsuperficiais, os pigmentos que mais influenciam a cor do solo é a hematita (cor vermelha) e goethita (cor amarela).

Os menores valores estimados para a altitude, carbono orgânico, argila e índice de avermelhamento para P1 (Figura 2) foram de 270,10 m, 9,04 g.kg-1, 22,13\% e 0,30, respectivamente, todos ocorrem numa distância de $691 \mathrm{~m}$ a partir do início do transecto. Estão relacionados à classe PAd, caracterizada por diferença textural entre os horizontes $\mathrm{A}$, menos argiloso, e o B com maior teor de argila (B textural Bt). Esta classe de solo está localizada na cota mais baixa do transecto, e apresenta caráter plíntico, indicativo de drenagem deficiente. Já, os maiores valores estimados foram $286 \mathrm{~m}$, 11,60 g.kg-1 $, 39,10 \%$ e 11,70 , respectivamente, os quais ocorreram na classe $\mathrm{LVd}$, caracterizada por solos profundos e intemperizados e bem drenados das cotas mais altas do transecto.

Tabela 2 - Modelos dos semivariogramas para os valores de altitude, carbono orgânico, teor de argila e índice de avermelhamento ao longo do transecto imaginário, nas profundidades de 0-20 e 40-60 cm.

\begin{tabular}{llllllll}
\hline Atributo & Profundidade $(\mathrm{cm})$ & Modelo & $\mathrm{C}_{0}$ & $\mathrm{C}_{0}+\mathrm{C}$ & $\mathrm{A}_{0}$ & $\mathrm{r}^{2}$ & SQR \\
\hline Altimetria $(\mathrm{m})$ & - & Esf. & 3,5 & 48 & 517 & 0,64 & 3092 \\
\hline $\begin{array}{l}\text { Carbono orgânico } \\
\left(\mathrm{g} . \mathrm{kg}^{-1}\right)\end{array}$ & $0-20$ & Esf. & 0,32 & 2,75 & 517 & 0,59 & 10,5 \\
\hline $\begin{array}{l}\text { Argila } \\
(\%)\end{array}$ & $40-60$ & Esf. & 0,25 & 2,62 & 517 & 0,63 & 9,94 \\
\hline \\
IAV & $0-20$ & Esf. & 9,26 & 33,28 & 223 & 0,59 & 263 \\
& $40-60$ & Esf. & 1,15 & 21,96 & 136 & 0,53 & 162 \\
\hline
\end{tabular}

Modelo de semivariograma: Esf. - Esférico, Exp. - Exponencial; $C_{0}$-Efeito pepita; $\left(C_{0}+C\right)$ - Patamar; $A_{0}$ - Alcance; $r^{2}$ - Coeficiente de regressão do semivariograma; SQR Soma dos Quadrados do Resíduo. 
Em P2, os menores valores estimados ocorreram a 652 $\mathrm{m}$ a partir do início do transecto. Os valores encontrados foram, respectivamente 2,51 g. $\mathrm{kg}^{-1}, 34,80 \%$ e 1,90 para carbono orgânico, argila e índice de avermelhamento, coincidindo com a classe dos Plintossolos (FFcd - solo com horizonte concrecionário dentro de $40 \mathrm{~cm}$ da superfície, com predominância de petroplintitas, e perfil imperfeitamente drenado). Ainda em P2, foram constatados que os maiores valores estimados para carbono orgânico $\left(6,41 \mathrm{~g} \cdot \mathrm{kg}^{-1}\right)$ e índice de avermelhamento $(12,00)$ ocorreram na classe $\mathrm{LVd}$, enquanto que o maior teor de argila $(49,60 \%)$ ocorreu no subsolo da classe FFcd.

A mínima amplitude de variação da altitude, representada pela largura mínima do intervalo de confiança, foi de 1,50 $\mathrm{m}$ e ocorreu na distância de $28 \mathrm{~m}$ do início do transecto, na classe LVd (Figura 2A). O carbono orgânico, a argila e o índice de avermelhamento em P1 apresentaram, como mínimas amplitudes de variação, os valores respectivos de $0,70 \mathrm{~g} \cdot \mathrm{kg}^{-1}$, $2,20 \%$ e 1,80, com variações mínimas para carbono orgânico, na classe LVAd e, para argila e índice de avermelhamento, na classe LVd. Enquanto que, em P2, carbono orgânico, argila e índice de avermelhamento apresentaram como mínima amplitude de variação os valores de 0,39 g. $\mathrm{kg}^{-1} ; 1,00 \%$ e 1,80, respectivamente, e a distância do início do transecto de 186 m para carbono orgânico (na classe LVd) e 242 m para argila e índice de avermelhamento (ambos na classe LVAd).

A largura máxima do intervalo de confiança para a altitude, que representa sua maior amplitude de variação, foi de 2,62 m e ocorreu a $418 \mathrm{~m}$ do inicio do transecto, na classe LVAd. Foram observados, em P1, os valores que representam a máxima amplitude de variação para carbono orgânico, conteúdo de argila e índice de avermelhamento de 0,75 g. $\mathrm{kg}^{-1}, 3,17 \%$ e 2,53, respectivamente. Enquanto que, em P2, o carbono orgânico, a argila e o índice de avermelhamento apresentaram a máxima amplitude de variação, com valores respectivos de $0,63 \mathrm{~g} \cdot \mathrm{kg}^{-1}, 3,40 \%$ e 2,53. Tanto em P1 como em P2, o máximo intervalo de confiança para os atributos estudados ocorreram dentro da classe LVAd.

\section{CONCLUSÕES}

A estimativa por krigagem ordinária do teor de argila, carbono orgânico, índice de avermelhamento e altitude ao longo de transecto, que cortou classes distintas de solo, permitiu observar que as maiores variabilidades espaciais dos atributos estudados ocorreram dentro da classe Latossolo Vermelho-Amarelo Distrófico, em função de sua ocorrência em diferentes segmentos da paisagem.

As classes Plintossolos e Argissolos (com caráter plíntico) têm ocorrência restrita às áreas com altimetrias estimadas inferiores a $280 \mathrm{~m}$; já, nas elevações superiores, ocorrem

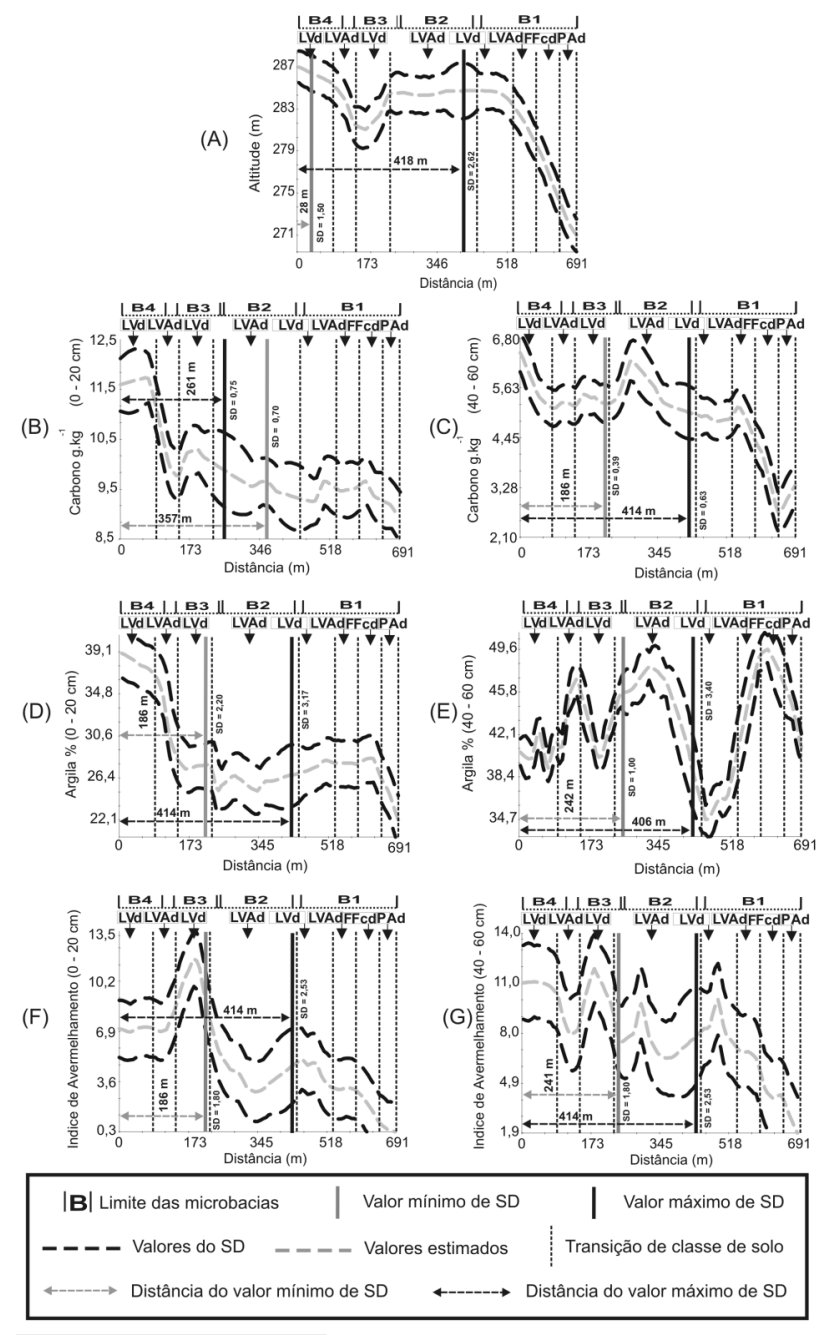

Figura 2 - Valores estimados por krigagem ordinária da altitude $(A)$, carbono orgânico (B e C), argila ( $\mathrm{D}$ e E) e índice de avermelhamento ( $\mathrm{F}$ e G), nas profundidades de 0-20 e 40-60 cm, ao longo do transecto na direção oesteleste.

exclusivamente Latossolos. Essas mudanças pedológicas em áreas tão pequenas parecem estar mais relacionadas ao relevo e material de origem do que aos outros fatores de formação do solo.

O índice de avermelhamento e a argila foram os atributos que mais variaram ao longo dos transectos, nas diferentes classes de solo. Isso demonstra a importância de se considerar as informações espaciais destes atributos em estudos de solo, principalmente nos casos em que a estatística clássica é insuficiente para a análise dos dados.

O índice de avermelhamento mostrou-se um bom indicador para identificar a drenagem dos solos. Neste estudo, os menores valores e a menor variabilidade espacial deste atributo foram encontrados nas áreas de pior drenagem. 


\section{BIBLIOGRAFIA CITADA}

Botschek, J.; Ferraz, J.; Jahnel, M.; Skowronek, A. 1996. Soil chemical properties of a toposequence under primary rain forest in the Itacoatiara vicinity (Amazonas, Brazil). Geoderma, 72: 119-132.

Brasil. 1980. Ministério das Minas e Energia. Secretaria Geral. Projeto RADAMBRASIL. Folha SC. 21 - Juruena. Rio de Janeiro, MMESG. 460pp. (Levantamento de Recursos Naturais, 20).

Cambardella, C.A.; Moorman, T.B.; Novak, J.M.; Parkin, T.B.; Karlen, D.L.; Turco, R.F.; Konopka, A.E. 1994. Field scale variability of soil properties in Central Iowa soils. Soil Sci. Soc. Am. J., 58: 1501-1511.

Carvalho, J.R.P. de, Silveira, P.M. da; Vieira, S.R. 2002. Geoestatística na determinação da variabilidade espacial de características químicas do solo sob diferentes preparos. Pesq. Agropec. Bras., 37(8): 1151-1159.

Couto, E.G.; Klamt, E. 1999. Variabilidade espacial de micronutrientes em solo sob pivô central no sul do Estado de Mato Grosso. Pesq. Agropec. Bras., 34(12): 2321-2329.

Couto, E.G.; Klamt, E.; Stein, A. 2000. Estimativa do teor de argila e do potássio trocável em solos esparsamente amostrados no sul do Estado de Mato Grosso. Revista Brasileira de Ciência do Solo, 24: 129-139.

Empresa Brasileira de Pesquisa Agropecuária. Centro Nacional de Pesquisa de Solos. 2 ed. 2006. Sistema brasileiro de classificação de solos. Rio de Janeiro, Embrapa/CNPS. 306pp.

Empresa Brasileira de Pesquisa Agropecuária. 1997. Manual de métodos de analise de solos. 2 ed. Rio de Janeiro, Embrapa/ CNPS. 212pp.

FAO/UNESCO. 1988. Soil map of the world: revised legend. World Soil Resources Report 60. Food and Agriculture Organization of the United Nations, Rome. 119pp.

Gonçalves, A.C.A.; Folegatti, M.V.; Vieira, S.R. 1999. Padrōes de amostragem e intensidade de krigagem na caracterização do armazenamento de água no solo, em área irrigada por pivô central. Revista Brasileira de Ciência do Solo, 23: 485-495.

Gonzalez, A.P.; Alves, M.C. 2005. Soil water storage and bulk density under three conditions of soil surface in a Cambisol of Lugo, Spain. Rev. Bras. Eng. Agric. Ambient., 9(1): 45-50.

Guimarães, E.C. 2001. Geoestatística básica e aplicada. Universidade Federal de Uberlândia. Uberlândia-MG. Faculdade de Matemática, Núcleo de estudos estatísticos e biométricos. 49pp.

IBGE. 1990. Projeto zoneamento das potencialidades dos recursos naturais da Amazônia Legal. Departamento de Recursos Naturais e Estudos Ambientais. Rio de Janeiro, IBGE. 212pp.

Jenny, H. 1941. Factors of soil formation: a system of quantitative pedology. MacGraw-Hill, New York. 281pp.

Johnson, M.S.; Lehmann, J.; Selva, E.C.; Abdo, M.; Riha, S.; Couto, E.G. 2004. Organic carbon fluxes within and exports from headwater catchments in the Southern Amazon. Cornell University, Ithaca, NY 14850, USA, 29pp.

Journel, A.C., Huijbregts, C.J. 1978. Mining geostatistics. Academic, London. 600pp.
Laurance, W.F.; Fearnside, P.M.; Laurance, S.G.; Delamonica, P.; Lovejoy, T.E.; Merona, J.M.R.; Chambers, J.Q.; Gascon, C. 1999. Relationship between soils and Amazon forest biomass: a landscape-scale study. Forest Ecology and Management, 118 : 127-138.

Lobato, L.A.O. 2000. Distribuição espacial de atributos pedológicos em áreas de cerrados mesotróficos no Pantanal de Poconé - MT. Dissertação de Mestrado, Universidade Federal de Mato Grosso, FAMEV, Cuiabá, Mato Grosso. 142pp.

Loureiro, V.R. 2002. Amazônia: uma história de perdas e danos, um futuro a (re)construir. Estud. Av., 16(45): 107-121. ISSN 0103-4014.

McBratney, A.B.; Webster, R. 1986. Choosing functions or semivariograms of soil properties and fitting them to sampling estimates. Journal Soil Science, 37: 617-639.

McGrath, D.A.; Smith, C.K.; Gholz, H.L.; Oliveira, F.A. 2001. Effects of land-use change on soil nutrient dynamics in Amazônia. Ecosystems, 4: 625-645.

Munsell Color Company. 1975. Munsell soil color charts. Baltimore.

Novaes Filho, J.P.; Selva, E.C.; Couto, E.G.; Lehmann, J.; Johnson, M.S.; Riha, S.J. 2007a. Distribuição espacial de carbono em solo sob floresta primária na Amazônia meridional. Revista Arvore, 31: 83-92.

Novaes Filho, J.P.; Couto, E.G.; Oliveira, V.A.D.; Johnson, M.S.; Lehmann, J.; Riha, S.J. 2007b. Variabilidade espacial de atributos físicos de solo usada na identificação de classes pedológicas de microbacias na Amazônia meridional. Revista Brasileira de Ciência do Solo, 31: 91-100.

Oliveira, F.H.T.; Novais, R.F.; Alvarez V., V.H.; Cantarutti, R.B.; Barros, N.F. 2000. Fertilidade do solo no sistema de plantio direto. In: Alvarez V., V.H; Schefer, C.E.G.R.; Barros, N.F.; Mello, J.W.V.; Costa, L.M. (Eds). Tópicos em ciências do solo. Vol. 2. Publicação da Sociedade Brasileira de Ciências do solo, Viçosa, Minas Gerais. p. 393-486.

Pimentel Gomes, F. 2000. Curso de estatística experimental. 14 ed. Degaspari, Piracicaba. 477pp.

Robertson, G.P. 2000. $G S^{+}$: Geostatistic for the enviroment sciences - GS+ user's guide version 5. Plainwell, Gamma Design Software. 200pp.

Rodrigues, L.C.M. 2005. Aspecto da variabilidade de atributos físicos e químicos do solo sob floresta primária. Dissertação de Mestrado, Universidade Federal de Mato Grosso, FAMEV, Cuiabá, Mato Grosso. 79pp.

Ruivo, M.L.P.; Quanz, B.; Baia, S.P.; Busseti, E.P.C.; Nagaishi, T.Y. 2002. The soils of the LBA experimental sites (Caxiuanã, Pará State, Brazil). In: 17 WCSS, Thailand. Anais eletrônicos (http://www.ldd.go.th/Wcss2002/papers/2152.pdf). Acesso: 20/10/2006

Soil Survey Staff. 1996. Soil Survey Laboratory Methods Manual. Soil Survey Investigations Report $\mathrm{n}^{\circ}$. 42, Version 3.0. U. S Department of Agriculture, Natural Resources Conservation Service, National Soil Survey Center, Washington, D.C. 663pp. 
Sombroek, W.G. 1984. Soils of the Amazon Region. In: Sioli, H.; Junk, W. (Eds). The Amazon limnology and landscape ecology of a mighty tropical river and its basin. Dordrecht/Boston/Lancaster. p. 521-536.

Statistical Package for the Social Sciences. 2000. SPSS for Windows. Base User's Guide, Release 10. Norusis, Marija J., SPSS Inc., Chicago, USA.

Surfer. 2002. Surfer 8.0: Contouring and 3d surface mapping for scientists and engineers. User's Guide, Golden Software Inc., New York, USA. 619pp.

Torrent, J.; Barròn, V. 1993. Laboratory measurement of soil color: theory and practice. In: SSSA. (Ed.). Soil Color. Special Publication, 31. Madison. p. 21-33.

Van Wambeke, A. 2002. Soils of the tropics: properties and appraisal. Second Edition. Cornell University, Department of Crop and Soil Sciences, Ithaca.
Vieira, S.R. 1997. Variabilidade espacial de argila, silte e atributos químicos em uma parcela experimental de um Latossolo Roxo de Campinas (SP). Bragantia, 56(1): 181-190.

Vieira, S.R. 2000. Geoestatística em estudos de variabilidade espacial do solo. In: Freitas, J.R. (Ed.). Tópicos em Ciências do Solo. Vol. 1. Publicação da Sociedade Brasileira de Ciências do solo, Viçosa, Minas Gerais. p. 1-54. ISSN 1519-3934.

Wilding, L.P.; Drees, L.R. 1983. Spatial variability and pedology. In: Wilding, L.P.; Smeck, N.E. (Eds.). Pedogenesis and Soil Taxonomy I: Concepts and Interactions. Amsterdam. p. 83-116.

Recebido em 21/01/2208

Aceito em 28/08/2008 